\title{
DYNAMICAL VERSIONS OF HARDY'S UNCERTAINTY PRINCIPLE: A SURVEY
}

\author{
AINGERU FERNÁNDEZ-BERTOLIN AND EUGENIA MALINNIKOVA
}

\begin{abstract}
The Hardy uncertainty principle says that no function is better localized together with its Fourier transform than the Gaussian. The textbook proof of the result, as well as one of the original proofs by Hardy, refers to the Phragmén-Lindelöf theorem. In this note we first describe the connection of the Hardy uncertainty to the Schrödinger equation, and give a new proof of Hardy's result which is based on this connection and the Liouville theorem. The proof is related to the second proof of Hardy, which has been undeservedly forgotten. Then we survey the recent results on dynamical versions of Hardy's theorem.
\end{abstract}

\section{INTRODUCTION}

There are many mathematical interpretations of the uncertainty principle, which states that the position and momentum of a quantum particle cannot be measured simultaneously, or that a signal cannot be well-localized both in time and in frequency. All of them refer to a double representation of a function; classically this is the function itself and its Fourier transform, though more recent versions of the uncertainty principle use some form of joint time-frequency representation, for example the short-time Fourier transform. Each uncertainty principle has an interesting and developing story, and in this note we tell only one of them.

The most famous uncertainty principle was introduced by Werner Heisenberg in 1927, and its mathematical formulation was given by Earle Hesse Kennard and Hermann Weyl shortly after. It says that

$$
\int_{\mathbb{R}^{d}}|x|^{2}|f(x)|^{2} \int_{\mathbb{R}^{d}}|\xi|^{2}|\widehat{f}(\xi)|^{2} \geq \frac{d^{2}}{4}\|f\|_{2}^{4}
$$

for all $f \in L^{2}\left(\mathbb{R}^{d}\right)$ or, equivalently,

$$
\int_{\mathbb{R}^{d}}|x|^{2}|f(x)|^{2}+\int_{\mathbb{R}^{d}}|\xi|^{2}|\widehat{f}(\xi)|^{2} \geq d \int_{\mathbb{R}^{d}}|f|^{2} .
$$

Received by the editors August 13, 2020.

2020 Mathematics Subject Classification. Primary 42A38, 35B05.

Key words and phrases. Uncertainty principle, Schrödinger equation.

The first author was partially supported by ERCEA Advanced Grant 2014669689 - HADE, by the project PGC2018-094528-B-I00 (AEI/FEDER, UE) and acronym "IHAIP", and by the Basque Government through the project IT1247-19.

The second author was partially supported by NSF grant DMS-1956294 and by the Research Council of Norway, project 275113. 
We always use the following normalization of the Fourier transform on $\mathbb{R}^{d}$,

$$
\widehat{f}(\xi)=\frac{1}{(2 \pi)^{d / 2}} \int_{\mathbb{R}^{d}} f(x) e^{-i x \cdot \xi} d x
$$

It is well-known that the Fourier transform is an isometry of $L^{2}\left(\mathbb{R}^{d}\right)$.

The equality in Heisenberg's uncertainty principle (10) is attained when $f$ is a generalized Gaussian function, i.e., $f(x)=\exp (-(A x, x))$, where $A$ is a positive definite matrix. The fact that the Gaussian is the best localized function in time and frequency was also recognized by English mathematician Godfrey H. Hardy in 1933, in the formulation of the uncertainty principle that now bears his name. Hardy attributed the remark that a function and its Fourier transform "cannot be very small" to Norbert Wiener and proved the following one dimensional result.

Theorem 1. Let $f \in L^{2}(\mathbb{R})$ satisfy $|f(x)| \leq C e^{-a|x|^{2}}$ and $|\widehat{f}(\xi)| \leq C e^{-b|\xi|^{2}}$. If $a b>1 / 4$, then $f=0$, and if $a b=1 / 4$, then $f(x)=c e^{-a|x|^{2}}$.

In his original article [28, Hardy gave two different proofs, and both refer to holomorphic functions and use some results of complex analysis. The first one employs the Phragmén-Lindelöf principle for entire functions. This proof or its variations can be found in many textbooks; see for example [29,40,42]. The second one also refers to entire functions but makes use of the Liouville theorem only (at least for the case when $a b>1 / 4$ ); it is more elementary and seems to be forgotten. We should also mention that Hardy proved a more general result, assuming that $|f(x)|=O\left(|x|^{m} e^{-a|x|^{2}}\right)$ and $|\widehat{f}(\xi)|=O\left(|\xi|^{m} e^{-b|\xi|^{2}}\right)$ as $x, \xi \rightarrow \pm \infty$, he showed that $f$ is a polynomial times $e^{-a|x|^{2}}$.

There was a search for a real variable proof of the Hardy uncertainty principle. A rather elementary (real variable) argument, given by Terence Tao in his book [43. §2.6], implies that $f$ is zero if in the statement above $a b>C_{0}$ for some large constant $C_{0}$. Another real variable proof for the case $a b>1$ is given by E. Pauwels and M. de Gosson in [39]. Surprisingly their proof employs prolate spheroidal wave functions, which, in the context of time frequency analysis, first appeared in the celebrated series of works of H. Landau, H. Pollak, and D. Slepian in the beginning of 1960s. The first complete real proof for the sharp result is given in [10].

Before we exhibit the main topic of this note, the dynamical interpretation of the Hardy uncertainty principle, and give a new proof of the result, we comment briefly on classical approaches and generalizations.

Hardy proved the theorem for the case $a=b=1 / 2$, which implies the general result by a simple rescaling. Gilbert W. Morgan gave the following generalization of Hardy's result already in 1934, 36].

Theorem 2. Let $1<p \leq 2$ and $1 / p+1 / q=1$. Suppose that $f \in L^{1}(\mathbb{R})$ and $|f(x)| \leq C e^{-a^{p}|x|^{p} / p}$ and $|\widehat{f}(\xi)| \leq C e^{-b^{q}|\xi|^{q} / q}$ and $a b>|\cos (p \pi / 2)|^{1 / p}$, then $f=0$.

For an interesting discussion of the Morgan theorem, extensions to functions that decay only along half-axes, and some remarkable related results, we refer the reader to [37] and 29].

The assumptions of both theorems formulated above are pointwise bounds for a function and its Fourier transform. In the 1980s M. Cowling and J. F. Price 
11] obtained versions where the bounds are replaced by an integral condition, the simplest version is the so-called $L^{2}$-Hardy uncertainty principle,

$$
e^{a|x|^{2}} f(x) \in L^{2}(\mathbb{R}) \quad \text { and } \quad e^{b|\xi|^{2}} \widehat{f}(\xi) \in L^{2}(\mathbb{R}),
$$

implying $f=0$ when $a b \geq 1 / 4$.

Hardy's theorem can be generalized to higher dimension, and the statement is exactly the same for $f \in L^{2}\left(\mathbb{R}^{d}\right)$. This can be deduced from the one dimensional result using the Radon transform; see 41. Note that we discuss only the simplest generalization of the Hardy uncertainty principle to $\mathbb{R}^{d}$. The appealing problem of natural higher dimensional statements is studied in [5, 6, 12, 13.

An interesting interpretation of Hardy's uncertainty principle was given in the beginning of the current century; see [9,15. It turns out that Theorem 1 is equivalent to the following statement.

Theorem 3. Let $u(t, x)$ be a solution to the free Schrödinger equation

$$
\partial_{t} u=i \Delta u(t, x) .
$$

Suppose that $u \in C^{1}\left([0, T], W^{2,2}\left(\mathbb{R}^{d}\right)\right)$ satisfies the decay conditions

$$
|u(0, x)| \leq C e^{-\alpha|x|^{2}} \quad \text { and } \quad|u(T, x)| \leq C e^{-\beta|x|^{2}},
$$

where $\alpha, \beta>0$.

(i) If $\alpha \beta>\left(16 T^{2}\right)^{-1}$, then $u(t, x)=0$.

(ii) If $\alpha \beta=\left(16 T^{2}\right)^{-1}$, then $u(t, x)=c e^{-(\alpha+i /(4 T))|x|^{2}}$.

A real-variable proof of this theorem is due to M. Cowling, L. Escauriaza, C. E. Kenig, G. Ponce, and L. Vega; see [10.

In this note we first show that the uniqueness result is equivalent to Hardy's theorem and give a simple proof of Theorem 3 . The proof involves holomorphic functions; however the proof of part (i) is based only on the Liouville theorem, which says that a bounded entire function is constant. The argument reminds one of the second proof of Theorem 1, given by Hardy in 28. The proof of part (ii) requires some analysis of a singular point of a holomorphic function. We then sketch the second proof of Hardy's theorem and give a relatively short and elementary proof of another uncertainty principle due to Beurling. The latter proof is inspired by the work of Hedenmalm; see [30]. To finish, we present an overview of the recent generalizations of Theorem 3 which are called the dynamical versions of Hardy's uncertainty principle.

\section{Free Schrödinger EQUATION}

2.1. Solution by the Fourier transform. In this section we present the classical formula for the solution of the Schrödinger equation, and we provide the details for the convenience of the reader. A generalization of the result is used later in the note. We consider the free Schrödinger equation

$$
\partial_{t} u(t, x)=i \Delta_{x} u(t, x),
$$

where $\Delta_{x}=\frac{\partial^{2}}{\partial x_{1}^{2}}+\cdots+\frac{\partial^{2}}{\partial x_{d}^{2}}$ is the Laplace operator. It is one of the simplest examples of a constant coefficient linear dispersive equation. Dispersive equations 
are called so since parts of solutions with different frequencies disperse with different speeds, spreading spatially. A plane wave is a solution to (2) of the form

$$
u_{\xi_{0}}(t, \xi)=\exp \left(i x \cdot \xi_{0}-i t\left|\xi_{0}\right|^{2}\right) .
$$

Clearly, any superposition of the plane waves is also a solution. The plane waves satisfy $|u(t, x)|=1$. Below we analyze solutions that decay in $x$. More precisely, we assume that $u \in C^{1}\left([0, T], W^{2,2}\left(\mathbb{R}^{d}\right)\right)$. This smoothness assumption can be weakened but we prefer to avoid the technical details in this note.

An effective method to solve linear constant coefficient dispersive equations is by applying the Fourier transform in spatial variables. Let $\widehat{u}(t, \xi)=\mathcal{F}_{x} u(t, x)$. Then (2) reads

$$
\partial_{t} \widehat{u}(t, \xi)=-i|\xi|^{2} \widehat{u}(t, \xi) .
$$

Thus the solution to (2) with initial data $u(0, x)=u_{0}(x) \in L^{2}\left(\mathbb{R}^{d}\right)$ satisfies

$$
\widehat{u}(t, \xi)=e^{-i t|\xi|^{2}} \widehat{u}_{0}(\xi) .
$$

Hence, by the Fourier inversion formula,

$$
\begin{aligned}
u(t, x) & =\frac{1}{(2 \pi)^{d / 2}} \int_{\mathbb{R}^{d}} e^{-i t|\xi|^{2}+i x \cdot \xi} \widehat{u}_{0}(\xi) d \xi \\
& =\frac{1}{(2 \pi)^{d}} \int_{\mathbb{R}^{d}} \int_{\mathbb{R}^{d}} e^{i\left(-t|\xi|^{2}+(x-y) \cdot \xi\right)} u_{0}(y) d y d \xi .
\end{aligned}
$$

The formula for $u(t, x)$ above can be written as the convolution

$$
u(t, x)=\int_{\mathbb{R}^{d}} u_{0}(y) K_{t}(x-y) d y,
$$

where $K_{t}$ is the (distributional) inverse Fourier transform of the function $e^{-i t|\xi|^{2}}$. Formally, we write

$$
K_{t}(x)=\frac{1}{(2 \pi)^{d}} \int_{\mathbb{R}^{d}} e^{i\left(t|\xi|^{2}+x \cdot \xi\right)} d \xi
$$

although the integral does not converge. To make sense of the integral, let

$$
K_{t}^{\varepsilon}(x)=\frac{1}{(2 \pi)^{d}} \int_{\mathbb{R}^{d}} e^{i\left(t|\xi|^{2}+x \cdot \xi\right)} e^{-\varepsilon|\xi|^{2}} d \xi .
$$

Then it is easy to see that

$$
K_{t}^{\varepsilon}(x)=\frac{1}{(4 \pi(\varepsilon+i t))^{d / 2}} e^{-|x|^{2} /(4(\varepsilon+i t))} .
$$

The limit of $K_{t}^{\varepsilon}(x)$ as $\varepsilon \rightarrow 0$ exists and is equal to

$$
K_{t}(x)=\frac{1}{(4 \pi i t)^{d / 2}} e^{-|x|^{2} /(4 i t)} .
$$

Therefore the solution to the Schrödinger equation is given by

$$
u(t, x)=\frac{1}{(4 \pi i t)^{d / 2}} \int_{\mathbb{R}^{d}} e^{i|x-y|^{2} /(4 t)} u_{0}(y) d y .
$$

We note that if $k_{t}$ denotes the standard heat kernel, then formally $K_{t}=k_{i t}$. 


\subsection{Uniqueness for the free Schrödinger evolution and Hardy's theorem.}

Using the integral formula for the solution (4), it is not difficult to see that Theorem 1 is equivalent to Theorem 3 with $d=1$. We show one implication: the Hardy uncertainty principle follows from the uniqueness result for the Schrödinger equation.

Assume that Theorem 3 is true, and let $f$ be a function as in the Hardy theorem. We define

$$
u(t, x)=\frac{1}{(4 \pi i t)^{1 / 2}} \int_{\mathbb{R}} e^{i|x-y|^{2} /(4 t)-i|y|^{2} / 4} f(y) d y,
$$

for $t>0$. Since $f$ is decaying fast, the function $u(t, x)$ is smooth. Then, differentiating the integrand, we see that $\partial_{t} u=i \Delta_{x} u$. Moreover, by taking the limit as $t \rightarrow 0$, we get $u(0, x)=e^{-i|x|^{2} / 4} f(x)$. Furthermore,

$$
u(1, x)=\frac{e^{i|x|^{2} / 4}}{(4 \pi i)^{1 / 2}} \widehat{f}(x / 2) .
$$

The assumptions in the Hardy theorem can now be translated to

$$
|u(0, x)| \leq C e^{-a|x|^{2}}, \quad|u(1, x)| \leq C e^{-b|x|^{2} / 4} .
$$

Now applying Theorem 3 with $T=1$, we conclude the argument.

The reverse implication can be shown in a similar way.

2.3. A proof of the uniqueness theorem. We now give a relatively elementary proof of Theorem 3 . The main idea is to consider the family of partial differential equations $\partial_{t} u=z \Delta_{x} u$ with complex parameter $z$. When $z= \pm 1$ we get the heat and the backward heat equations, while $z=i$ corresponds to the Schrödinger equation. Computations, similar to ones presented in Section 2.1] show that the fundamental solution is

$$
k_{t}(z)(x)=(4 \pi z t)^{-d / 2} e^{-|x|^{2} /(4 z t)} .
$$

Thus for a fast decaying initial condition $u_{0}(x)$, the solution to the equation is given by $u(t, x)=u_{0} * k_{t}(z)$, so $k_{t}(z)=: k_{t z}$ is a complex extension of the heat kernel.

Assume now that

$$
\left|u_{0}(x)\right|=|u(0, x)| \leq e^{-\alpha|x|^{2}} .
$$

We start with the initial condition $u(0, x)=u_{0}(x)$ that decays fast, and we solve the generalized heat equation. We see that the heat equation itself is solvable (it corresponds to $z$ real and positive) as is the Schrödinger equation (corresponding to pure imaginary $z$ ), but the backward heat equation cannot be solved in general, and our function is not defined for small real negative $z$. We consider the function

$$
F(z, x)=\frac{1}{(4 \pi z)^{d / 2}} \int_{\mathbb{R}^{d}} e^{-|x-y|^{2} /(4 z)} u_{0}(y) d y=k_{z} * u_{0},
$$

for $\Omega_{0}=\{z \in \mathbb{C}: \Re(-1 /(4 z))-\alpha<0\}$. Solving the last inequality for $z$, we see that the integral above converges uniformly on compact subsets of the domain

$$
\Omega_{0}=\{z \in \mathbb{C}:|z+1 /(8 \alpha)|>1 / 8 \alpha\} .
$$

The function $F^{2}(z, x)$ is a holomorphic function of $z$ in $\Omega_{0}$, when $x \in \mathbb{R}^{d}$ is fixed. Note that we take the square of $F$ to avoid the branching of $\sqrt{z}$.

Now, we start with $u(T, x)=u_{1}(x)$ and define

$$
G(z, x)=k_{z-i T} * u_{1}=\frac{1}{(4 \pi(z-i T))^{d / 2}} \int_{\mathbb{R}^{d}} e^{-|x-y|^{2} /(4(z-i T))} u_{1}(y) d y .
$$




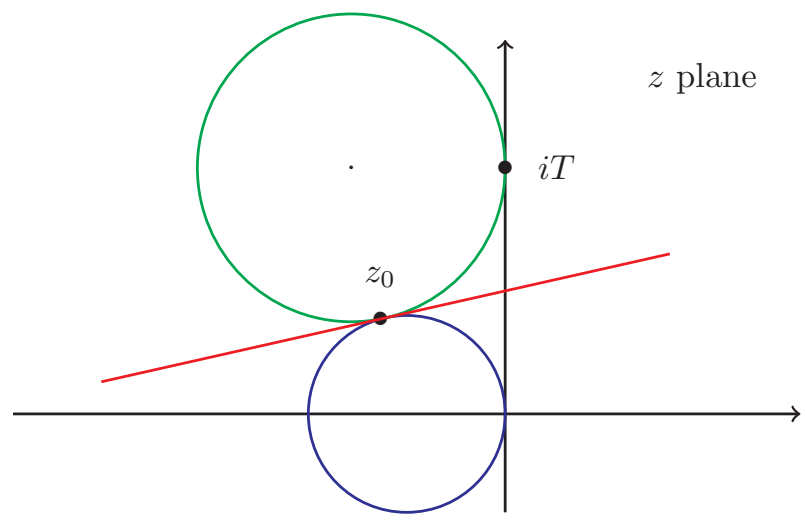

Figure 1. Tangent circles $\partial \Omega_{0}$ and $\partial \Omega_{1}$ and their common tangent line $l$ for the case $A B=T^{2} / 4, z$-plane

Using the decay of $u_{1}$, we see that $G^{2}(z, x)$ is well defined and holomorphic in the domain

$$
\Omega_{1}=\{z \in \mathbb{C}:|z-i T+1 / 8 \beta|>1 /(8 \beta)\} .
$$

Moreover, $G(i t, x)=u(t, x)$ when $t \in(0, T)$. Hence the holomorphic functions $F^{2}(\cdot, x)$ and $G^{2}(\cdot, x)$ coincide on the interval $(0, T)$. Therefore $F^{2}(\cdot, x)$ is extended to a holomorphic function on $\Omega_{0} \cup \Omega_{1}$.

To simplify the notation, we denote $(8 \alpha)^{-1}=A$ and $(8 \beta)^{-1}=B$. Then the complements of $\Omega_{0}$ and $\Omega_{1}$ are circles with the radii $A$ and $B$, while the distance between the centers is $\sqrt{T^{2}+(A-B)^{2}}$.

If $A B<T^{2} / 4$ (which is equivalent to $16 \alpha \beta>T^{-2}$ ), then the circles do not intersect. Thus $F^{2}(z, x)$ extends to an entire function in $z$ for each fixed $x$. It also satisfies

$$
\begin{aligned}
\left|F^{2}(z, x)\right| & \leq \frac{C}{(4 \pi|z|)^{d}}\left(\int_{\mathbb{R}^{d}} e^{-\Re\left(|x-y|^{2} /(4 z)\right)} e^{-\alpha|y|^{2}} d y\right)^{2} \\
& =\frac{C}{(4|z|(\alpha+\gamma))^{d}} e^{-2 \gamma \alpha|x|^{2} /(\gamma+\alpha)}
\end{aligned}
$$

where $\gamma=\Re(1 /(4 z))$. We fix $x$ and note that $F^{2}(z, x)$ is uniformly bounded as $|z|>1 / \alpha$. Then, by the Liouville theorem, $F^{2}(z, x)$ is a constant function in $z$ for each $x$. This means that $\partial_{t} u=0$ and thus $\Delta u=0$. There are no nonzero decaying harmonic functions, therefore $u(t, x)=0$.

This proof of Theorem 3(i) uses only the facts that the function $e^{c z}$ satisfies the mean value property and that a bounded function satisfying the mean value property on the whole plane is a constant. An elementary proof of the latter can be found in 38 .

Now assume that $16 \alpha \beta=T^{-2}$, i.e., $A B=T^{2} / 4$. Then the circles $\partial \Omega_{0}$ and $\partial \Omega_{1}$ touch at one point, which we denote by $z_{0}$; see Figure 1 Thus $F^{2}(z, x)$ is a holomorphic function in $\mathbb{C} \backslash\left\{z_{0}\right\}$. We consider $x=0$ and claim that $F^{2}(z, 0)$ has a pole at $z_{0}$. To prove that, we draw the common tangent line $l$ to the circles $\partial \Omega_{0}$ and $\partial \Omega_{1}$, and we consider the images of this line under the transformations $\zeta=z^{-1}$ and $\eta=(z-i T)^{-1}$. These are circles $\omega_{0}$ and $\omega_{1}$ passing through the origin, while the images of the circles $\partial \Omega_{0}$ and $\partial \Omega_{1}$ under those two respective transformations 


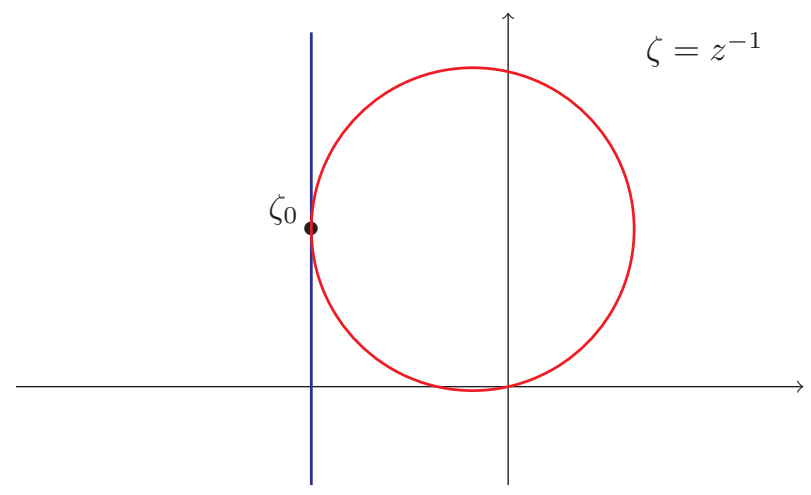

Figure 2. Circle $\omega_{0}$ and tangent line $l_{0}$ in $\zeta=1 / z$-plane

are vertical lines $l_{0}$ and $l_{1}$ tangent to $\omega_{0}$ and $\omega_{1}$; see Figure 2. We see that $\omega_{0}$ is defined by the equation

$$
\Re\left(\zeta-\zeta_{0}\right)=\left|\zeta-\zeta_{0}\right|^{2} /\left(2 r_{0}\right),
$$

where $\zeta_{0}=z_{0}^{-1}$ and $r_{0}$ is the radius of $\omega_{0}$. Let $z$ be a point close to $z_{0}$ lying above the line $l$ (on the other side of the line $l$ than $\partial \Omega_{0}$ ). Then $\zeta=z^{-1}$ lies inside the disk bounded by $\omega_{0}$, and we have the following inequality

$$
\Re\left(\zeta-\zeta_{0}\right) \geq c\left|\zeta-\zeta_{0}\right|^{2} \geq c_{1}\left|z-z_{0}\right|^{2},
$$

where $c=\left(2 r_{0}\right)^{-1}$ and $c_{1}=c\left|z_{0}\right|^{-4} / 2$. The estimate (5) implies

$$
\left|F^{2}(z, 0)\right| \leq C\left|z-z_{0}\right|^{-2 d}
$$

when $z$ is in the half-plane above the line $l$. For the other half-plane we repeat the argument, using the function $G^{2}$, and conclude that $F^{2}(z, 0)$ has a pole at $z_{0}$ of order less than or equal to $2 d$.

Similarly, we consider the functions

$$
\begin{aligned}
F_{j}(z, x) & =\partial F(x, z) / \partial x_{j} \\
& =\frac{1}{2 z(4 \pi z)^{d / 2}} \int_{\mathbb{R}^{d}} e^{-|x-y|^{2} /(4 z)}\left(y_{j}-x_{j}\right) u_{0}(y) d y, \quad j=1, \ldots, d .
\end{aligned}
$$

Then each $F_{j}^{2}(z, x)$ extends to a holomorphic function in $\mathbb{C} \backslash\left\{z_{0}\right\}$ and $F_{j}(z, 0)$ has a pole at $z_{0}$. An estimate of $F_{j}(z, 0)$ gives

$$
\left|F_{j}^{2}(z, 0)\right| \leq C\left|z-z_{0}\right|^{-2 d-1} \text {. }
$$

Finally, consider $\zeta=\zeta_{0}+t$, where $t>0$ is real and small. For this case the inequality (6) can be replaced by $\Re\left(\zeta-\zeta_{0}\right)=\left|\zeta-\zeta_{0}\right|$. Then, repeating the argument above and taking $z=1 / \zeta$, we see that $\left(z-z_{0}\right)^{d} F^{2}(z, 0)$ and $\left(z-z_{0}\right)^{d+1} F_{j}^{2}(z, 0)$ are bounded along the curve $z=z_{0}\left(1+t z_{0}\right)^{-1}, t>0$. Thus $F^{2}(z, 0)$ has a pole at $z_{0}$ of order not exceeding $d$, while for each $F_{j}^{2}(z, 0), j=1, \ldots, d$, the order of this pole does not exceed $d+1$.

We assume first that $d=1$. To finish the proof of the endpoint case, we use the Hermite functions,

$$
\psi_{n}(t)=e^{t^{2} / 2} \frac{\partial^{n}}{\partial t^{n}} e^{-t^{2}}=H_{n}(t) e^{-t^{2} / 2}
$$


which form an orthogonal basis for $L^{2}(\mathbb{R})$. More generally, for any complex number $\gamma$ with $\Re \gamma>0$, we may define the generalized Hermite functions

$$
\psi_{n}^{(\gamma)}(t)=H_{n}(\sqrt{2 \gamma} t) e^{-\gamma t^{2}},
$$

which still form an orthogonal basis for $L^{2}(\mathbb{R})$.

First we consider $F^{2}(z, 0)$. This is a holomorphic function in $\mathbb{C} \backslash\left\{z_{0}\right\}$ that tends to zero at infinity and has a simple pole at $z_{0}$, thus

$$
F^{2}(z, 0)=b\left(z-z_{0}\right)^{-1} \text {. }
$$

Hence

$$
\int_{-\infty}^{\infty} e^{-y^{2} /(4 z)} u_{0}(y) d y=c z^{1 / 2}\left(z-z_{0}\right)^{-1 / 2}
$$

A simple computation shows that

$$
\int_{-\infty}^{\infty} e^{-y^{2} /(4 z)} e^{-\gamma y^{2}} d y=2 \sqrt{\pi} \frac{z^{1 / 2}}{(1+4 z \gamma)^{1 / 2}} .
$$

We choose $\gamma=-1 /\left(4 z_{0}\right)=-\zeta_{0} / 4$ and see that for some constant $c_{0}$ and every $\zeta$

$$
c_{0} \int_{-\infty}^{\infty} e^{-y^{2} \zeta / 4} e^{-\gamma y^{2}} d y=\int_{-\infty}^{\infty} e^{-y^{2} \zeta / 4} u_{0}(y) d y .
$$

This means that all even moments of $u_{0}$ are equal to the corresponding moments of $c_{0} e^{-\gamma|y|^{2}}$, and thus $u_{0}(y)+u_{0}(-y)=2 c_{0} e^{-\gamma y^{2}}$.

Then, similarly, we consider $F_{1}^{2}(z, 0)$. We have

$$
F_{1}^{2}(z, 0)=b_{2}\left(z-z_{0}\right)^{-2}+b_{1}\left(z-z_{0}\right)^{-1} .
$$

On the other hand

$$
\int_{0}^{\infty} y^{k} e^{-y^{2} /(4 z)} e^{-\gamma y^{2}} d y=c_{k} \frac{z^{(k+1) / 2}}{(1+4 z \gamma)^{(k+1) / 2}} .
$$

Representing $y u_{0}(y)$ as the series in $\psi_{n}^{(\gamma)}$, we conclude that

$$
y u_{0}(y)-y u_{0}(-y)=2\left(c_{1}+c_{2} y\right) e^{-\gamma y^{2}} .
$$

Now, taking $y \rightarrow 0$ and using that $u_{0}(y)=G(0, y)$ is a continuous function, we see that $c_{1}=c_{2}=0$. Thus $u_{0}$ is even and $u_{0}(y)=c_{0} e^{-\gamma|y|^{2}}$. It is not difficult to check that $\gamma=\alpha+i / 4 T$. This concludes the proof of Theorem 3 for the case $d=1$.

To complete the proof in higher dimensions, we consider $F(z, x)$ and all its partial derivatives in the spatial variables at $x=0$. Rewriting the integral in polar coordinates, we have

$$
F(z, 0)=\frac{1}{(4 \pi z)^{d / 2}} \int_{0}^{\infty} r^{d-1} \int_{S^{d-1}} u_{0}\left(r y^{\prime}\right) d \sigma\left(y^{\prime}\right) e^{-r^{2} /(4 z)} d r .
$$

Let $\Phi(r)=r^{d-1} \int_{S^{d-1}} u_{0}\left(r y^{\prime}\right) d \sigma\left(y^{\prime}\right)$. The identity (8) and the fact that $F^{2}$ has a pole at $z_{0}=-1 /(4 \gamma)$ of order not exceeding $d$ imply that

$$
\Phi(r)=\sum_{l=0}^{d-1} c_{l} r^{l} e^{-\gamma r^{2}}
$$


Moreover, since $\Phi(r)$ has a zero of order $d-1$ at zero, we conclude that $\Phi(r)=$ $c r^{d-1} e^{-\gamma r^{2}}$. On the other hand, looking at the partial derivatives of $F$, we see that for any homogeneous polynomial $p(y)$ of degree $k$,

$$
\Phi_{p}(r)=r^{d-1+k} \int_{S^{d-1}} p\left(y^{\prime}\right) u_{0}\left(r y^{\prime}\right) d \sigma\left(y^{\prime}\right)
$$

is a linear combination of the form $\sum_{0}^{d-1+k} c_{l} r^{l} e^{-\gamma r^{2}}$. If $\int_{S^{d-1}} p\left(y^{\prime}\right) d \sigma\left(y^{\prime}\right)=0$, then $\Phi_{p}(r)=0$ since its zero at the origin is of order larger than $d-1+k$. Therefore $u_{0}$ is orthogonal to all polynomials with zero mean on each sphere centered at the origin. This implies that $u_{0}$ is a constant on each such sphere and thus $u_{0}(y)=c e^{-\gamma|y|^{2}}$.

2.4. Heat equation. We saw that the Schrödinger equation and the heat equation are close relatives. Therefore, it is natural that the Hardy uncertainty principle implies a uniqueness result for the heat equation.

Theorem 4. Let $u(t, x) \in C^{1}\left([0, T], W^{2,2}\left(\mathbb{R}^{d}\right)\right)$ be a solution to the heat equation $\partial_{t} u=\Delta_{x} u$. Suppose that $u(0, x) \in L^{1}\left(\mathbb{R}^{d}\right)$ and $|u(T, x)| \leq e^{-\delta|x|^{2}}$. If $\delta \geq 1 /(4 T)$, then $u=0$.

The case $\delta=1 / 4 T$ corresponds to the situation $u(0, x)$ is the Dirac delta function. The fact that the Hardy uncertainty principle implies Theorem 4 follows by applying the Fourier transform in variable $x$, which gives

$$
\widehat{u}(t, \xi)=e^{-t|\xi|^{2}} \widehat{u}(0, \xi) .
$$

Thus, if the initial data $u_{0}(x)=u(0, x) \in L^{1}\left(\mathbb{R}^{d}\right)$, then $|\widehat{u}(T, \xi)| \leq C e^{-T|\xi|^{2}}$. Combined with the decay condition for $u(T, x)$, it implies that $u(T, x)=0$ if $\delta>$ $1 /(4 T)$ and $u(T, x)=c_{0} e^{-\delta|x|^{2}}$ if $\delta=1 /(4 T)$. The latter implies $\widehat{u}_{0}(\xi)=c$ and $u$ is a multiple of the Dirac delta function.

We can also prove Theorem 4 using the approach suggested in the previous section. The condition $|u(T, x)| \leq e^{-\delta|x|^{2}}$ implies that the function

$$
\tilde{G}^{2}(z, x)=\left(k_{z-T} * u(T, x)\right)^{2}
$$

is holomorphic in the domain

$$
\tilde{\Omega}=\left\{z \in \mathbb{C}:\left|z-T+(8 \delta)^{-1}\right|>(8 \delta)^{-1}\right\},
$$

while the condition $u(0, x) \in L^{1}$ implies that the function

$$
\tilde{F}^{2}(z, x)=\left(k_{z} * u(0, x)\right)^{2}
$$

is holomorphic when $\Re(z)>0$. Moreover, we know that $\tilde{F}^{2}(t, x)=\tilde{G}^{2}(t, x)$ when $t \in(0, T)$. If $\delta>1 /(4 T)$, the two domains cover the whole complex plane, and we obtain a bounded entire function. It leads to a contradiction in the same way as above for the Schrödinger equation. If $\delta=1 /(4 T)$, then the resulting function is holomorphic in $\mathbb{C} \backslash\{0\}$, but the singularity at 0 is removable for almost every $x$ since

$$
\lim _{z \rightarrow 0} \tilde{F}^{2}(z, x)=u^{2}(0, x)
$$

almost everywhere. And we get a contradiction again.

We also note that Theorem 4 does not imply the limit case $(a b=1 / 4)$ in the Hardy uncertainty principle. The reason is that in general a bounded function is not a Fourier transform of an $L^{1}$-function. To obtain an equivalent statement, one 
should extend the notion of solutions of the heat equation to the case when the initial data is a measure.

\section{The SECOnd Proof of Hardy and Beurling's unCERTAinty PRINCIPle}

3.1. On the forgotten proof of Hardy. We were not able to find the second proof of Hardy or its variations in any textbook, so we give a sketch of this proof here as pointed at in the introduction, for the cone $a=b=\frac{1}{2}$. First, Hardy notes that the decay conditions on $f$ and $\widehat{f}$ imply the decay conditions on $f_{e}(x)=(f(x)+f(-x)) / 2$ and $f_{o}=(f(x)-f(-x)) / 2$ and their Fourier transforms. Next, the functions $f_{1}=\left(f_{e}+\widehat{f}_{e}\right) / 2, f_{2}=\left(f_{e}-\widehat{f}_{e}\right) / 2, f_{3}=\left(f_{o}+i \widehat{f}_{o}\right) / 2$, and $f_{4}=\left(f_{0}-i \widehat{f}_{o}\right) / 2$ also satisfy the decay condition together with the Fourier transforms. So one may assume that $\widehat{f}=i^{k} f$.

Let first $f$ be even, so that $\widehat{f}= \pm f$. Hardy considers the function

$$
\lambda_{f}(s)=\int_{0}^{\infty} e^{-s x^{2} / 2} f(x) d x,
$$

where $f$ decays as the Gaussian. Then $\lambda_{f}$ is a holomorphic function when $\Re(s)>-1$ and the equation $\widehat{f}= \pm f$ translates into the identity

$$
\lambda_{f}(s)=s^{-1 / 2} \lambda_{f}(1 / s) .
$$

We skip the details of choosing the right branch of the root function here.

Then the function $\mu(s)=\sqrt{s+1} \lambda_{f}(s)$ satisfies $\mu(s)=\mu(1 / s)$ and it can be extended to a holomorphic function in $\mathbb{C} \backslash\{-1\}$. Moreover, $\mu$ has a pole at $s_{0}=-1$. Finally, Hardy refers to the injectivity of the transform, i.e., $\lambda_{f}=\lambda_{g}$ if and only if $f=g$, and the identity for the Hermite functions

$$
\int_{0}^{\infty} \psi_{2 n}(t) e^{-s t^{2} / 2} d t=c_{n} \frac{(s-1)^{n}}{(s+1)^{n+1 / 2}} .
$$

The case where $f$ is odd is not written down in [28. For this case we suggest considering the function

$$
\tilde{\lambda}_{f}(s)=\int_{0}^{\infty} x e^{-s x^{2} / 2} f(x) d x= \pm \sqrt{\frac{2}{\pi}} \int_{0}^{\infty} x e^{-s x^{2} / 2} \int_{0}^{\infty} f(y) \sin x y d y d x
$$

where the second identity follows from the fact $f= \pm i \widehat{f}$. Then $\tilde{\lambda}_{f}(s)=s^{-3 / 2} \tilde{\lambda}_{f}(1 / s)$. As before, we consider $\mu(s)=\sqrt{(s+1)} \tilde{\lambda}_{f}(s)$ that satisfies $\mu(s)=s^{-1} \mu(1 / s)$. This function extends to a holomorphic function in $\mathbb{C} \backslash\{-1\}$ such that $|\mu(s)| \rightarrow 0$ when $|s| \rightarrow \infty$. Further, $\mu$ has a pole at $s_{o}=-1$, and one concludes the argument by the same techniques of the even case.

3.2. Beurling's uncertainty principle. The following version of the uncertainty principle is due to Arne Beurling

Theorem 5. Suppose that $f \in L^{2}(\mathbb{R})$ and

$$
\int_{\mathbb{R}} \int_{\mathbb{R}} e^{|x \xi|}|f(x)||\widehat{f}(\xi)| d x d \xi<\infty .
$$

Then $f=0$. 
The theorem appeared in the collected works of Beurling in [4] and dates back to the 1960s. The original proof of Beurling uses the Phragmén-Lindelöf theorem and it can be found in [31. Higher dimensional versions of the Beurling theorem were obtained in [6]. In 2012 Håkan Hedenmalm gave another proof and generalized the statement in [30]. His result was further extended in [26]. We follow the ideas in [30. to give a relatively short proof of the original statement of Beurling. Clearly, the Beurling theorem implies the $L^{2}$-version of the Hardy uniqueness result.

First, by taking the real and imaginary parts of $f$ we may reduce the problem to the case when $f$ is real-valued. Now, following the idea of Hedenmalm, consider the function

$$
F(s)=\int_{\mathbb{R}} \int_{\mathbb{R}} e^{i s x \xi} f(x) \widehat{f}(\xi) d x d \xi .
$$

Then $F$ is well-defined and holomorphic in the strip $S=\{s \in \mathbb{C}:|\Im(s)|<1\}$. Moreover, by the monotone convergence theorem, $F$ is continuous on $\bar{S}$. For real $s$, we have

$$
F(s)=\sqrt{\pi / 2} \int_{\mathbb{R}} f(x) f(s x) d x
$$

we have used that $f, \widehat{f} \in L^{1}(\mathbb{R})$. Then $F(s)=s^{-1} F(1 / s)$ for $s \in \mathbb{R} \backslash\{0\}$. We obtain that $F$ can be extended to a holomorphic function on $\mathbb{C} \backslash\{ \pm i\}$. The singularities at $s= \pm i$ are removable since the function is continuous at these points. Finally, the functional equation $F(s)=s^{-1} F(1 / s)$ and the fact that $F$ is bounded near the origin imply that $|F(s)| \rightarrow 0$ when $|s| \rightarrow \infty$. Thus $F=0$. In particular,

$$
F(1)=\sqrt{\frac{\pi}{2}} \int_{\mathbb{R}} f^{2}(x) d x=0 .
$$

Finally, since $f$ is real-valued, we conclude that $f=0$.

\section{ReCEnt Versions of the Uniqueness theorem}

We now return to the dynamical versions of the uncertainty principles. In the last 15 years the uniqueness results for the free Schrödinger and heat equations were generalized to a large class of evolutions. We give an overview of some of these results in this section.

4.1. Schrödinger and heat equations with a potential. First, we consider the Schrödinger equation with a potential,

$$
\partial_{t} u(t, x)=i(\Delta u+V u) .
$$

In a series of articles, Luis Escauriaza, Carlos E. Kenig, Gustavo Ponce, and Luis Vega (15 19) generalized the uniqueness result for the case when $V$ is a bounded potential satisfying one of the following conditions:

(i) $\lim _{R \rightarrow \infty} \int_{0}^{T} \sup _{|x|>R}|V(t, x)| d t=0$,

(ii) $V(t, x)=V_{1}(x)+V_{2}(t, x)$,

where $V_{1}$ is real-valued (and does not depend on $t$ ) and $V_{2}$ satisfies, for some positive $\alpha$ and $\beta$,

$$
\sup _{[0, T]}\left\|e^{\alpha \beta T^{2}|x|^{2} /(\sqrt{\alpha} t+\sqrt{\beta}(T-t))^{2}} V_{2}(t)\right\|_{L^{\infty}\left(\mathbb{R}^{n}\right)}<+\infty .
$$


Theorem 6. Let $u \in C\left([0, T], L^{2}\left(\mathbb{R}^{d}\right)\right)$ be a solution to [10), where $V$ satisfies either (i) or (ii). If $|u(0, x)| \leq C e^{-\alpha|x|^{2}}$ and $|u(T, x)| \leq C e^{-\beta|x|^{2}}$ with $\alpha \beta>$ $1 /\left(16 T^{2}\right)$, then $u=0$.

Note that the condition on $\alpha \beta$ is sharp! The result is further generalized to semilinear equations and covariant Schrödinger evolution in [18] and [3, 8], and to Navier-Stokes equation in 14 .

We outline the proof of Theorem [6. First it suffices to consider the case when $\alpha=\beta$; the Appell transform reduces the general case to this one. We renormalize the solution and assume that $T=1$. The first step is to show logarithmic convexity of some weighted norm of the solution. The method can be compared to the one used by Shmuel Agmon for elliptic equations in 1960s; see [1. For each $t \in[0,1]$ and $\xi \in S^{d-1}$, we define

$$
H(t)=\int_{\mathbb{R}^{d}}\left|e^{\mu|x+R b(t) \xi|^{2}} u(t, x)\right|^{2} d x
$$

where $b(t)=16 \mu t(1-t)$. The derivative of $v(t, x)=e^{\mu|x+R b(t) \xi|^{2}} u(t, x)$ in $t$ is written as the sum of a symmetric and antisymmetric operator,

$$
\partial_{t} v=(S+A) v
$$

Then a straightforward calculation implies that

$$
(\log H(t))^{\prime \prime} \geq 2\langle(S A-A S) v, v\rangle .
$$

Careful estimates on $S A-A S$ show that $(\log H(t))^{\prime \prime} \geq-16 \mu R^{2}-C_{v}$, where $C_{v}$ denotes a constant that depends on the potential. Therefore

$$
H(t) \exp \left(-32 \mu R^{2} t(1-t)\right) \leq C_{v} H(0)^{1-t} H(1)^{t} .
$$

The right-hand side does not depend on $R$, while in the left-hand side for $t=1 / 2$ the weight (with which $u^{2}$ is integrated) is

$$
\exp \left(2 \mu|x+4 \mu R \xi|^{2}-8 \mu R^{2}\right) .
$$

We look at the coefficient in front of $R^{2}$ : if $32 \mu^{3}>8 \mu$, it is positive, and thus we see that $u(1 / 2, x)=0$ for almost each $x$ by letting $R \rightarrow \infty$. Then $u \equiv 0$. This formal computation can be justified if $H(0)$ and $H(1)$ are finite. This proves Theorem 6 when $\alpha=\beta>1 / 2$.

To extend the result for the range $\alpha=\beta>1 / 4$, Escauriaza, Kenig, Ponce, and Vega developed an ingenious bootstrapping argument. To sketch their argument, we write (11) as

$$
\int_{\mathbb{R}^{d}}|u(t, x)|^{2} e^{2 \mu|x|^{2}+4 R \mu b(t) x \cdot \xi-2 R^{2} b(t)(1-\mu b(t))} d x \leq C_{v} H(0)^{1-t} H(1)^{t} .
$$

Under the assumption $\alpha=\beta \leq 1 / 2$, a formal integration of the last inequality with respect to $R$ leads to

$$
\int_{\mathbb{R}^{d}}|u(t, x)|^{2} e^{2 a_{1}(t)|x|^{2}} d x \leq C_{v} H(0)^{1-t} H(1)^{t}
$$

for $a_{1}(t)=\mu /(1-\mu b(t))$. Notice that $a_{1}(1 / 2-t)=a_{1}(1 / 2+t), a_{1}(0)=a_{1}(1)=\mu$, and $a_{1}(t)>\mu$ when $t \in(0,1)$, which shows that the solution $u$ decays faster at 
$(0,1)$ than at the endpoints. Next, one can construct a positive function $b_{1}(t)$ such that $b_{1}(0)=b_{1}(1)=0$ and so that

$$
H_{1}(t)=\int_{\mathbb{R}^{d}}\left|e^{a_{1}(t)\left|x+R b_{1}(t) \xi\right|^{2}} u(t, x)\right|^{2} d x
$$

satisfies

$$
H_{1}(t) \exp \left(-2 R^{2} b_{1}(t)\right) \leq C_{v} H_{1}(0)^{1-t} H_{1}(1)^{t}=C_{v} H(0)^{1-t} H(1)^{t} .
$$

Note that this is again (11) but $\mu$ and $b$ are replaced by $a_{1}$ and $b_{1}$. A similar study as before tells us that $1-a_{1}(1 / 2) b_{1}(1 / 2) \leq 0$ implies $u \equiv 0$, while otherwise we can integrate again to improve the decay at $(0,1)$. This self-improvement can be repeated several times, resulting in a sequence of functions

$$
a_{k+1}(t)=\frac{a_{k}(t)}{1-a_{k}(t) b_{k}(t)}, a_{0}(t)=\mu
$$

such that

$$
\mu<a_{1}(t)<\cdots<a_{k}(t), \quad t \in(0,1) .
$$

On each step the new function satisfies $a_{k}(1 / 2-t)=a_{k}(t+1 / 2), a_{k}(0)=a_{k}(1)=\mu$, and

$$
\left\|e^{a_{k}(t)|x|^{2}} u(t, x)\right\|_{2}^{2} \leq H(0)^{1-t} H(1)^{t} .
$$

As for the functions $b_{k}(t)$, they are constructed from $a_{k}(t)$ in such a way that at each step relation (12) is satisfied for the pair of functions $a_{k}$ and $b_{k}$. More precisely, as shown in 17, $b_{k}(t)$ is the solution to

$$
\left\{\begin{array}{l}
\ddot{b}_{k}=-\frac{1}{a_{k}^{2}}\left(\ddot{a}_{k}+32 a_{k}^{3}-\frac{3\left(\dot{a}_{k}\right)^{2}}{2 a_{k}}\right), \\
b_{k}(0)=b_{k}(1)=0 .
\end{array}\right.
$$

If, for some $k$, we have $1-a_{k}(1 / 2) b_{k}(1 / 2) \leq 0$, which translates in a condition on parameter $\mu$, the iterative argument stops and we reach a contradiction implying $u \equiv 0$. Otherwise, the process is infinite and the limit function $a(t)=\lim _{k \rightarrow \infty} a_{k}(t)$ exists. Since (13) implies $b_{k}(t)=\left(a_{k+1}-a_{k}\right) /\left(a_{k} a_{k+1}\right)$, the functions $b_{k}$ will converge to 0 and, from the differential equation satisfied by $b_{k}$, one can deduce that the limit function $a(t)$ satisfies

$$
\left\{\begin{array}{l}
\ddot{a}+32 a^{3}-\frac{3(\dot{a})^{2}}{2 a}=0, \\
a(0)=a(1)=\mu .
\end{array}\right.
$$

Solving the ordinary differential equation under the constraint $a(1 / 2-t)=$ $a(1 / 2+t)$ leads to

$$
a(t)=\frac{C}{4\left(1+(t-1 / 2)^{2} C^{2}\right)}
$$

for some $C>0$. Computing the maximum in $C$ of $\mu=a(0)=C /\left(4+C^{2}\right)$, we see that $\mu$ must be less than $1 / 4$. Then Theorem 6 follows.

A similar strategy gives a powerful generalization of Theorem 4, see [19].

Theorem 7. Let $V(t, x) \in L^{\infty}\left(\mathbb{R} \times \mathbb{R}^{d}\right)$ and $u$ be a solution to the equation

$$
\partial_{t} u=\Delta_{x} u+V u,
$$

$\left.u \in L^{\infty}\left([0, T], L^{2}\left(\mathbb{R}^{d}\right)\right) \cap L^{2}\left([0, T], H^{1}\left(\mathbb{R}^{d}\right)\right]\right)$. If $|u(T, x)| \leq e^{-\delta|x|^{2}}$ and $\delta>1 / \sqrt{T}$, then $u=0$. 
A natural question is what decay a stationary solution to the Schrödinger equation may have. The question was asked by E. M. Landis in 1960 (see [7, 32]), who conjectured that if $V \in L^{\infty}\left(\mathbb{R}^{d}\right), \Delta u+V u=0$ in $\mathbb{R}^{d}$, and $|u(x)| \leq C \exp \left(-|x|^{1+c}\right)$ with $c>0$, then $u \equiv 0$. The conjecture was disproved by V. Z. Meshkov in 36, who constructed an example of a complex valued $u$ and $V$ such that $|u(x)| \leq$ $\exp \left(-|x|^{4 / 3}\right)$ and proved that there are no solution with a faster decay. A remaining question is whether the Landis conjecture holds under the assumption that $V$ is real valued. In spite of some recent progress [33, this is an open problem in dimensions $d \geq 3$.

4.2. Discrete evolutions. Another twist of the uniqueness results for Schrödinger equation was given in 20, 21, 23, 27, where uniqueness theorems are obtained for the discrete equation. Let $\Delta_{d}$ be the usual discrete Laplacian on $\mathbb{Z}^{d}$. We consider the equation

$$
\partial_{t} U(t, n)=i\left(\Delta_{d} U(t, n)+V(t, n) U(t, n)\right),
$$

where $n \in \mathbb{Z}^{d}$ and $V$ is a bounded potential. The uniqueness results say that a solution to the discrete Schrödinger equation which decays fast at two times is trivial. To find the optimal decay, we consider the free evolution with $V=0$. In dimension $d=1$, there is a solution $U_{0}(t, n)=i^{-n} e^{-2 i t} J_{n}(1-2 t)$, where $J_{n}$ is the Bessel function, and it has optimal decay at $t=0$ and $t=1$. The role of the Gaussian is now played by the Bessel function. This fact is related to different behavior of the heat kernels: for the continuous case the standard heat kernel is $k(1, x)=(4 \pi)^{-1 / 2} \exp \left(-x^{2} / 4\right)$, while for the discrete case the heat kernel is $K(1, n)=e^{-1}\left|I_{n}(1)\right| \asymp e^{-1}\left(n ! 2^{n}\right)^{-1}$, where $I_{n}$ are the modified Bessel functions, $I_{n}(z)=(-i)^{n} J_{n}(i z)$.

Theorem 8. Let $U(t, n)$ be a solution to (14), with $V \equiv 0$, on $[0,1] \times \mathbb{Z}$. Suppose that

$$
|U(0, n)|+|U(1, n)| \leq \frac{C}{\sqrt{|n|}}\left(\frac{e}{2|n|}\right)^{|n|}, \quad n \in \mathbb{Z} \backslash\{0\} .
$$

Then $U(t, n)=C i^{-n} e^{-2 i t} J_{n}(1-2 t)$. In particular, a solution to the free discrete Schrödinger equation cannot decay faster than $J_{n}(1)$ both at $t=0$ and $t=1$.

The idea of the proof is to consider the function $\psi(t, z)=\sum_{-\infty}^{\infty} U(t, n) z^{n}$. It is not difficult to show that it is defined on the unit circle $|z|=1$, Moreover, the decay of $U(0, l)$ and $U(1, l)$ shows that $\psi(0, z)$ and $\psi(1, z)$ are entire functions. Equation (14) implies

$$
\psi(t, z)=e^{i\left(z+z^{-1}-2\right) t} \psi(0, z),
$$

and $\psi(t, z)$ extends to an entire function for any $t \in[0,1]$. Careful analysis of this function and application of the Phragmén-Lindelöf theorem finishes the proof. It would be interesting to find a real-variable, or at least more elementary, proof.

This result was generalized to special classes of time-independent potentials. General bounded potentials were considered in [27] (in dimension $d=1$ ) and [23] (in arbitrary dimension). The result is as follows.

Theorem 9. Let $U(t, n) \in C^{1}\left([0,1]: \ell^{2}\left(\mathbb{Z}^{d}\right)\right)$ be a solution to (14) on $[0,1] \times \mathbb{Z}^{d}$. Suppose that $\|V\|_{\infty} \leq 1$. There exists constant $\gamma$ such that if

$$
|U(0, n)|+|U(1, n)| \leq C \exp (-\gamma|n| \log |n|), \quad n \in \mathbb{Z}^{d} \backslash\{0\},
$$

then $U=0$. 
The approach in 27] follows the scheme of [18] described in the first step of the proof of Theorem 6] in Section 4.1, We describe the details of [23. The idea is to make use of the following result, known in the literature as Carleman-type inequality, whose proof relies on the computation of a commutator between a symmetric and an antisymmetric operator. In what follows, $\|\cdot\|_{2}$ stands for $\|\cdot\|_{L^{2}\left([0,1], \ell^{2}\left(\mathbb{Z}^{d}\right)\right)}$ and $\|\cdot\|_{\infty}$ will represent the supremum norm.

Lemma 1. Let $\varphi:[0,1] \rightarrow \mathbb{R}$ be a smooth function, and let $\gamma>\frac{\sqrt{d}}{2}$. There exist $R_{0}=R_{0}\left(d,\left\|\varphi^{\prime}\right\|_{\infty}+\left\|\varphi^{\prime \prime}\right\|_{\infty}, \gamma\right)$ and $c=c\left(d,\left\|\varphi^{\prime}\right\|_{\infty}+\left\|\varphi^{\prime \prime}\right\|_{\infty}\right)$ such that if $R>R_{0}$, $\alpha \geq \gamma R \log R$ and $g \in C_{0}^{1}\left([0,1], \ell^{2}\left(\mathbb{Z}^{d}\right)\right)$ has its support contained in the set

$$
\left\{(t, n):\left|n / R+\varphi(t) e_{1}\right| \geq 1\right\}
$$

then

$$
\sqrt{\sinh \left(2 \alpha / R^{2}\right)} \sinh (2 \alpha / \sqrt{d} R)\left\|e^{\alpha\left|\frac{n}{R}+\varphi(t) e_{1}\right|^{2}} g\right\|_{2} \leq c\left\|e^{\alpha\left|\frac{n}{R}+\varphi(t) e_{1}\right|^{2}}\left(i \partial_{t}+\Delta_{d}\right) g\right\|_{2} .
$$

Thanks to this inequality, one can deduce lower bounds for nontrivial solutions of (14) with a general bounded potential. In order to do that, consider the cut-off functions,

$$
\theta^{R}(x)=\left\{\begin{array}{l}
1,|x| \leq R-1, \\
0,|x| \geq R,
\end{array} \quad \mu(x)=\left\{\begin{array}{l}
1,|x| \geq 2, \\
0,|x| \leq 1,
\end{array} \quad \varphi(t)=\left\{\begin{array}{l}
3, t \in\left[\frac{3}{8}, \frac{5}{8}\right], \\
0, t \in\left[0, \frac{1}{4}\right] \cup\left[\frac{3}{4}, 1\right],
\end{array}\right.\right.\right.
$$

and define $g(t, n)=U(t, n) \theta^{R}(n) \mu\left(\frac{n}{R}+\varphi(t) e_{1}\right)$. By means of the Leibniz rule and carefully studying the size of the weight $e^{\alpha\left|\frac{n}{R}+\varphi(t) e_{1}\right|^{2}}$ in the support of the derivatives of the cut-off functions, one can check that

$$
\begin{aligned}
& \sqrt{\sinh \left(2 \alpha / R^{2}\right)} \sinh (2 \alpha / \sqrt{d} R)\left\|e^{\alpha\left|\frac{n}{R}+\varphi e_{1}\right|^{2}} g\right\|_{2} \\
& \quad \leq c\left\|e^{\alpha\left|\frac{n}{R}+\varphi e_{1}\right|^{2}}\left(i \partial_{t}+\Delta_{d}\right) g\right\|_{2} \\
& \quad \leq c\left(\left\|e^{\alpha\left|\frac{n}{R}+\varphi e_{1}\right|^{2}} g\right\|_{2}+e^{16 \alpha} \lambda(R)+e^{4 \alpha}\|U\|_{2}\right),
\end{aligned}
$$

where $\lambda(R)=\left(\int_{0}^{1} \sum_{R-2 \leq|n| \leq R+1}|U(t, n)|^{2}\right)^{1 / 2}$. The fact that $\alpha$ needs to be larger than $\gamma R \log R$ implies that for $R \geq R_{0}$, depending only on the dimension, the first term in the right-hand side can be absorbed in the left-hand side (one can check that the product of sinh functions increases with $R$ ). On the other hand, if we assume $\int_{1 / 2-1 / 8}^{1 / 2+1 / 8}|U(t, 0)|^{2} d t \geq 1$, the norm in the left-hand side is bounded by

$$
\left\|e^{\alpha\left|\frac{n}{R}+\varphi e_{1}\right|^{2}} g\right\|_{2} \geq e^{9 \alpha}
$$

since $g(t, 0)=U(t, 0)$ if $t \in[1 / 2-1 / 8,1 / 2+1 / 8]$, and in that region the weight is exactly $e^{9 \alpha}$. So for $R \geq R_{0}$ depending on $\|U\|_{2}$, the last term in the right-hand side of (15) can also be absorbed, and we get

$$
c\left(\int_{0}^{1} \sum_{R-2 \leq|n| \leq R+1}|U(t, n)|^{2}\right)^{1 / 2} \geq e^{-5 \alpha}=e^{-c R \log R}
$$

after choosing $\alpha$ appropriately. This proves the following lower bound. 
Theorem 10. Let $U \in C^{1}\left([0,1]: \ell^{2}\left(\mathbb{Z}^{d}\right)\right)$ satisfy (14). Assume that

$$
\int_{0}^{1} \sum_{n \in \mathbb{Z}^{d}}|U(t, n)|^{2} d t \leq A^{2}, \int_{1 / 2-1 / 8}^{1 / 2+1 / 8}|U(t, 0)|^{2} d t \geq 1
$$

and

$$
\|V\|_{\infty}=\sup _{t \in[0,1], j \in \mathbb{Z}^{d}}\{|V(t, n)|\} \leq 1 .
$$

Then there exist $R_{0}=R_{0}(d, A)>0$ and $c=c(d)$ such that for $R \geq R_{0}$ it follows that

$$
\lambda(R) \equiv\left(\int_{0}^{1} \sum_{R-2 \leq|n| \leq R+1}|U(t, n)|^{2}\right)^{1 / 2} \geq c e^{-c R \log R} .
$$

We remark that this lower bound only uses the fact that the solution is nontrivial and that the constant $c$ in front of the term $R \log R$ only depends on the dimension.

Theorem 10 implies Theorem 9. The decay conditions at times $t=0$ and $t=1$ imply upper bounds for the term $\lambda(R)$. Indeed, monotonicity results from [23, 27] show that

$$
\left\|e^{\gamma|n| \log |n|} U(0)\right\|_{\ell^{2}\left(\mathbb{Z}^{d}\right)}+\left\|e^{\gamma|n| \log |n|} U(1)\right\|_{\ell^{2}\left(\mathbb{Z}^{d}\right)}<\infty
$$

for some fixed $\gamma$ implies $\left\|e^{\gamma|n| \log |n|} U(t)\right\|_{\ell^{2}\left(\mathbb{Z}^{d}\right)}<\infty$ for all $t \in[0,1]$. Hence, if (16) is satisfied,

$$
\lambda(R) \leq C e^{-\gamma R \log R}
$$

for a positive constant $C$. Thus, by letting $R$ tend to infinity, we arrive to a contradiction if $\gamma$ is large enough, since the upper bound decays faster than the lower bound, and therefore $U \equiv 0$ if (16) is satisfied for $\gamma>\gamma_{0}$ where $\gamma_{0}$ depends only on the dimension. However, these results are not sharp. We know that the bound can be improved to $\exp (-|n|(\log |n|+\mu))$ for some large constant $\mu$. For the free equation $(V=0)$, the condition $\mu>\log 2-1$ implies the uniqueness, and the question is whether for bounded potential the uniqueness result holds with the same range of $\mu$.

Further uniqueness results for solutions of discrete Schrödinger type equations, inspired by the works of Escauriaza, Kenig, Ponce, and Vega on the continuous case, can be found in $[2,22,25,34]$.

\section{ABout the AUthors}

Aingeru Fernández-Bertolin is assistant professor at the University of the Basque Country, in Spain. His field of research is partial differential equations, studying unique continuation properties for PDEs in different settings.

Eugenia Malinnikova is professor of mathematics at Stanford University and is adjunct professor at Norwegian University of Science and Technology. She got her $\mathrm{PhD}$ in St. Petersburg, Russia, under the supervision of Victor Havin.

\section{REFERENCES}

[1] S. Agmon, Unicité et convexité dans les problèmes différentiels (French), Séminaire de Mathématiques Supérieures, No. 13 (Été, 1965), Les Presses de l’Université de Montréal, Montreal, Que., 1966. MR 0252808

[2] I. Álvarez-Romero, Uncertainty principle for discrete Schrödinger evolution on graphs, Math. Scand. 123 (2018), no. 1, 51-71, DOI 10.7146/math.scand.a-105369. MR 3843554 
[3] J. A. Barceló, L. Fanelli, S. Gutiérrez, A. Ruiz, and M. C. Vilela, Hardy uncertainty principle and unique continuation properties of covariant Schrödinger flows, J. Funct. Anal. 264 (2013), no. 10, 2386-2415, DOI 10.1016/j.jfa.2013.02.017. MR3035060

[4] A. Beurling, The collected works of Arne Beurling. Vol. 2: Harmonic analysis, Contemporary Mathematicians, Birkhäuser Boston, Inc., Boston, MA, 1989. Edited by L. Carleson, P. Malliavin, J. Neuberger and J. Wermer. MR.1057614

[5] A. Bonami and B. Demange, A survey on uncertainty principles related to quadratic forms, Collect. Math. Vol. Extra (2006), 1-36. MR2264204

[6] A. Bonami, B. Demange, and P. Jaming, Hermite functions and uncertainty principles for the Fourier and the windowed Fourier transforms, Rev. Mat. Iberoamericana 19 (2003), no. 1, 23-55, DOI 10.4171/RMI/337. MR1993414

[7] J. Bourgain and C. E. Kenig, On localization in the continuous Anderson-Bernoulli model in higher dimension, Invent. Math. 161 (2005), no. 2, 389-426, DOI 10.1007/s00222-004-0435-7. MR2180453

[8] B. Cassano and L. Fanelli, Sharp Hardy uncertainty principle and Gaussian profiles of covariant Schrödinger evolutions, Trans. Amer. Math. Soc. 367 (2015), no. 3, 2213-2233, DOI 10.1090/S0002-9947-2014-06383-6. MR3286512

[9] S. Chanillo, Uniqueness of solutions to Schrödinger equations on complex semi-simple Lie groups, Proc. Indian Acad. Sci. Math. Sci. 117 (2007), no. 3, 325-331, DOI 10.1007/s12044007-0028-7. MR 2352052

[10] M. Cowling, L. Escauriaza, C. E. Kenig, G. Ponce, and L. Vega, The Hardy uncertainty principle revisited, Indiana Univ. Math. J. 59 (2010), no. 6, 2007-2025, DOI 10.1512/iumj.2010.59.4395. MR2919746

[11] M. Cowling and J. F. Price, Generalisations of Heisenberg's inequality, Harmonic analysis (Cortona, 1982), Lecture Notes in Math., vol. 992, Springer, Berlin, 1983, pp. 443-449, DOI 10.1007/BFb0069174. MR729369

[12] M. A. de Gosson, Two geometric interpretations of the multidimensional Hardy uncertainty principle, Appl. Comput. Harmon. Anal. 42 (2017), no. 1, 143-153, DOI 10.1016/j.acha.2015.11.002. MR 3574565

[13] B. Demange, Uncertainty principles and light cones, J. Fourier Anal. Appl. 21 (2015), no. 6, 1199-1250, DOI 10.1007/s00041-015-9401-6. MR3421916

[14] Z. Duan, S. Han, and P. Sun, On unique continuation for Navier-Stokes equations, Abstr. Appl. Anal., posted on 2015, Art. ID 597946, 16, DOI 10.1155/2015/597946. MR3335432

[15] L. Escauriaza, C. E. Kenig, G. Ponce, and L. Vega, On uniqueness properties of solutions of Schrödinger equations, Comm. Partial Differential Equations 31 (2006), no. 10-12, 1811-1823, DOI 10.1080/03605300500530446. MR2273975

[16] L. Escauriaza, C. E. Kenig, G. Ponce, and L. Vega, Hardy's uncertainty principle, convexity and Schrödinger evolutions, J. Eur. Math. Soc. (JEMS) 10 (2008), no. 4, 883-907, DOI 10.4171/JEMS/134. MR2443923

[17] L. Escauriaza, C. E. Kenig, G. Ponce, and L. Vega, The sharp Hardy uncertainty principle for Schrödinger evolutions, Duke Math. J. 155 (2010), no. 1, 163-187, DOI 10.1215/001270942010-053. MR2730375

[18] L. Escauriaza, C. E. Kenig, G. Ponce, and L. Vega, Uniqueness properties of solutions to Schrödinger equations, Bull. Amer. Math. Soc. (N.S.) 49 (2012), no. 3, 415-442, DOI 10.1090/S0273-0979-2011-01368-4. MR2917065

[19] L. Escauriaza, C. E. Kenig, G. Ponce, and L. Vega, Hardy uncertainty principle, convexity and parabolic evolutions, Comm. Math. Phys. 346 (2016), no. 2, 667-678, DOI 10.1007/s00220015-2500-z. MR 3535897

[20] A. Fernández-Bertolin, A discrete Hardy's uncertainty principle and discrete evolutions, J. Anal. Math. 137 (2019), no. 2, 507-528, DOI 10.1007/s11854-019-0002-1. MR 3938012

[21] A. Fernández-Bertolin, Convexity properties of discrete Schrödinger evolutions, J. Evol. Equ. 20 (2020), no. 1, 257-278, DOI 10.1007/s00028-019-00524-6. MR4072656

[22] A. Fernández-Bertolin and P. Jaming, Uniqueness for solutions of the Schrödinger equation on trees, Ann. Mat. Pura Appl. (4) 199 (2020), no. 2, 681-708, DOI 10.1007/s10231-01900896-z. MR 4079656 
[23] A. F. Bertolin and L. Vega, Uniqueness properties for discrete equations and Carleman estimates, J. Funct. Anal. 272 (2017), no. 11, 4853-4869, DOI 10.1016/j.jfa.2017.03.006. MR 3630642

[24] A. Fernández-Bertolin and J. Zhong, Hardy's uncertainty principle and unique continuation property for stochastic heat equations, ESAIM Control Optim. Calc. Var. 26 (2020), Paper No. 9, 22, DOI 10.1051/cocv/2019009. MR4064470

[25] A. Fernández-Bertolin, A. Grecu, and L. I. Ignat, Hardy uniqueness principle for the linear Schrödinger equation on quantum regular trees, arXiv:2005.06204 (2020).

[26] X. Gao, On Beurling's uncertainty principle, Bull. Lond. Math. Soc. 48 (2016), no. 2, 341348, DOI 10.1112/blms/bdw006. MR3483071

[27] P. Jaming, Y. Lyubarskii, E. Malinnikova, and K.-M. Perfekt, Uniqueness for discrete Schrödinger evolutions, Rev. Mat. Iberoam. 34 (2018), no. 3, 949-966, DOI 10.4171/RMI/1011. MR3850274

[28] G. H. Hardy, A Theorem Concerning Fourier Transforms, J. London Math. Soc. 8 (1933), no. 3, 227-231, DOI 10.1112/jlms/s1-8.3.227. MR1574130

[29] V. Havin and B. Jöricke, The uncertainty principle in harmonic analysis, Ergebnisse der Mathematik und ihrer Grenzgebiete (3) [Results in Mathematics and Related Areas (3)], vol. 28, Springer-Verlag, Berlin, 1994. MR 1303780

[30] H. Hedenmalm, Heisenberg's uncertainty principle in the sense of Beurling, J. Anal. Math. 118 (2012), no. 2, 691-702, DOI 10.1007/s11854-012-0048-9. MR3000695

[31] L. Hörmander, A uniqueness theorem of Beurling for Fourier transform pairs, Ark. Mat. 29 (1991), no. 2, 237-240, DOI 10.1007/BF02384339. MR1150375

[32] V. A. Kondratiev and E. M. Landis, Qualitative theory of second-order linear partial differential equations (Russian), 1988. MR1133457

[33] A. Logunov, E. Malinnikova, N. Nadirashvili, and F. Nazarov, The Landis conjecture on exponential decay, arXiv:2007.07034 (2020).

[34] Y. Lyubarskii and E. Malinnikova, Sharp uniqueness results for discrete evolutions, Nonlinear partial differential equations, mathematical physics, and stochastic analysis, EMS Ser. Congr. Rep., Eur. Math. Soc., Zürich, 2018, pp. 423-436. MR3823854

[35] V. Z. Meshkov, On the possible rate of decay at infinity of solutions of second order partial differential equations, Math. USSR Sbornik 72 (1992), 343-360.

[36] G. W. Morgan, A Note on Fourier Transforms, J. London Math. Soc. 9 (1934), no. 3, 187192, DOI 10.1112/jlms/s1-9.3.187. MR1574180

[37] F. L. Nazarov, Local estimates for exponential polynomials and their applications to inequalities of the uncertainty principle type (Russian, with Russian summary), Algebra i Analiz 5 (1993), no. 4, 3-66; English transl., St. Petersburg Math. J. 5 (1994), no. 4, 663-717. MR 1246419

[38] E. Nelson, A proof of Liouville's theorem, Proc. Amer. Math. Soc. 12 (1961), 995, DOI 10.2307/2034412. MR259149

[39] E. Pauwels and M. de Gosson, On the prolate spheroidal wave functions and Hardy's uncertainty principle, J. Fourier Anal. Appl. 20 (2014), no. 3, 566-576, DOI 10.1007/s00041-0149319-4. MR 3217488

[40] B. Simon, Harmonic analysis, A Comprehensive Course in Analysis, Part 3, American Mathematical Society, Providence, RI, 2015. MR 3410783

[41] A. Sitaram, M. Sundari, and S. Thangavelu, Uncertainty principles on certain Lie groups, Proc. Indian Acad. Sci. Math. Sci. 105 (1995), no. 2, 135-151, DOI 10.1007/BF02880360. MR 1350473

[42] S. Thangavelu, An introduction to the uncertainty principle: Hardy's theorem on Lie groups, Progress in Mathematics, vol. 217, Birkhäuser Boston, Inc., Boston, MA, 2004. With a foreword by Gerald B. Folland. MR 2008480

[43] T. Tao, An epsilon of room, I: real analysis: Pages from year three of a mathematical blog, Graduate Studies in Mathematics, vol. 117, American Mathematical Society, Providence, RI, 2010. MR2760403 
Universidad del País Vasco /Euskal Herriko Unibertsitatea (UPV/EHU), Dpto. de Matemáticas, Apartado 644, 48080 Bilbao, Spain

Email address: aingeru.fernandez@ehu.eus

Department of Mathematics, Stanford University, Stanford, California; and Department of Mathematical Sciences, Norwegian University of Science and Technology, TrondheIM, NORWAY

Email address: eugeniam@stanford.edu 